IJMMS 2003:4, 199-207

PII. S016117120320627X

http://ijmms.hindawi.com

(c) Hindawi Publishing Corp.

\title{
NONLINEAR VARIATIONAL INEQUALITIES ON REFLEXIVE BANACH SPACES AND TOPOLOGICAL VECTOR SPACES
}

\author{
ZEQING LIU, JEONG SHEOK UME, and SHIN MIN KANG
}

Received 8 June 2002

\begin{abstract}
The purpose of this paper is to introduce and study a class of nonlinear variational inequalities in reflexive Banach spaces and topological vector spaces. Based on the KKM technique, the solvability of this kind of nonlinear variational inequalities is presented. The obtained results extend, improve, and unify the results due to Browder, Carbone, Siddiqi, Ansari, Kazmi, Verma, and others.
\end{abstract}

2000 Mathematics Subject Classification: 47J20, 49J40.

1. Introduction. It is well known that variational inequality theory has significant applications in various fields of mathematics, physics, economics, and engineering science. Siddiqi et al. [6] considered the solvability of a class of nonlinear variational inequality problems in nonempty closed convex subsets and nonempty compact convex subsets of reflexive Banach spaces and locally convex spaces, respectively. Carbone [3] extended the results of Siddiqi et al. [6]. Recently, Verma [7] presented the existence and uniqueness of solutions for a class of nonlinear variational inequality problems involving a combination of operators of $p$-monotone and $p$-Lipschitz types, which generalizes a result due to Browder [1]. On the other hand, Carbone [4] established the existence of solutions of a class of nonlinear variational inequality problems in nonempty convex subsets of topological vector spaces.

Inspired and motivated by research works [1, 3, 4, 6, 7], in this paper, we study the solvability of a new class of nonlinear variational inequality problems in nonempty closed convex subsets and nonempty convex subsets of reflexive Banach spaces and topological vector spaces, respectively. The obtained results extend, improve, and unify the corresponding results in $[1,3,4,6,7]$ and others.

2. Preliminaries. Throughout this paper, let $\mathbb{R}=(-\infty,+\infty)$ and $\mathbb{R}^{+}=[0,+\infty)$. Let $\left\langle X, X^{*}\right\rangle$ be a dual system of topological vector spaces or of Banach spaces, let the bilinear form $\langle\cdot, \cdot\rangle$ be continuous, and let $K$ be a nonempty convex set in $X$. Let $A, B, C: K \rightarrow X^{*}, M: X^{*} \times X^{*} \times X^{*} \rightarrow X^{*}$, and $h: K \times K \rightarrow K$ be mappings and let $f: K \rightarrow \mathbb{R}$ be a convex functional. Assume that $h$ is linear with respect to the first argument. We consider the nonlinear variational inequality problem: 
for each given $w \in X^{*}$, find $u \in K$ such that

$$
\langle M(A u, B u, C u)-w, h(v, u)\rangle+f(v)-f(u) \geq 0 \quad \forall v \in K
$$

If $M(x, y, z)=x-y+z$, then problem (2.1) is equivalent to the following problem: for each given $w \in X^{*}$, find $u \in K$ such that

$$
\langle A u-B u+C u-w, h(v, u)\rangle+f(v)-f(u) \geq 0 \quad \forall v \in K .
$$

REMARK 2.1. For a suitable choice of $M, A, B, C, h, w$, and $f$, problem (2.1) includes a few kinds of known variational inequalities as special cases (see $[1,3,4,6,7]$ and the references therein).

Recall that a multivalued mapping $F: X \rightarrow 2^{X}$ is called the KKM mapping if, for every finite subset $\left\{u_{i}: 1 \leq i \leq n\right\}$ of $X$, $\operatorname{conv}\left\{u_{i}: 1 \leq i \leq n\right\} \subset \cup_{i=1}^{n} F u_{i}$, where $\operatorname{conv}(A)$ denotes the convex hull of $A$.

LEMMA 2.2 [5]. Let $K$ be a nonempty subset of a Hausdorff topological vector space $X$ and let $F: K \rightarrow 2^{X}$ be a KKM mapping. If $F x$ is closed in $X$ for any $x \in K$ and there exists at least a point $u \in K$ such that $F u$ is compact, then

$$
\bigcap_{x \in K} F x \neq \varnothing
$$

LEMMA 2.3 [2]. Let $C$ be a nonempty convex subset of a topological vector space $X$. Let $A \subset C \times C$ and $g: C \rightarrow C$ such that the following conditions are satisfied:

(i) $(x, g x) \in A$ for all $x \in C$;

(ii) for each $y \in C$, the set $\{x \in C:(x, g y) \notin A\}$ is convex or empty;

(iii) for each $x \in C$, the set $\{y \in C:(x, g y) \in A\}$ is closed in $C$;

(iv) there exists a nonempty compact convex subset $B$ of $C$ such that the set $D=\{y \in C:(x, g y) \in A$ for all $x \in B\}$ is compact.

Then, there exists $u \in C$ satisfying $C \times\{g u\} \subset A$.

In the rest of this section, let $(X,\|\cdot\|)$ be a Banach space, $X^{*}$ the topological dual space of $X$, and $\langle\cdot, \cdot\rangle$ the dual pair between $X$ and $X^{*}$.

Definition 2.4. A mapping $A: K \rightarrow X^{*}$ is called $h-\phi$ monotone with respect to the first argument of $M: X^{*} \times X^{*} \times X^{*} \rightarrow X^{*}$ if there exist mappings $\phi: \mathbb{R}^{+} \rightarrow \mathbb{R}^{+}$with $\phi(0)=0, h: K \times K \rightarrow K$ satisfying

$$
\langle M(A v, \cdot, \cdot)-M(A u, \cdot, \cdot), h(v, u)\rangle \geq \phi(\|h(v, u)\|) \quad \forall v, u \in K
$$


Definition 2.5. A mapping $A: K \rightarrow X^{*}$ is called $h-\phi$ monotone if there exist mappings $\phi: \mathbb{R}^{+} \rightarrow \mathbb{R}^{+}$with $\phi(0)=0, h: K \times K \rightarrow K$ satisfying

$$
\langle A v-A u, h(v, u)\rangle \geq \phi(\|h(v, u)\|) \quad \forall v, u \in K
$$

DEFINITION 2.6. A mapping $B: K \rightarrow X^{*}$ is called $h-\psi$ relaxed monotone with respect to the second argument of $M: X^{*} \times X^{*} \times X^{*} \rightarrow X^{*}$ if there exist mappings $\psi: \mathbb{R}^{+} \rightarrow \mathbb{R}^{+}$with $\psi(0)=0, h: K \times K \rightarrow K$ satisfying

$$
\langle M(\cdot, B v, \cdot)-M(\cdot, B u, \cdot), h(v, u)\rangle \geq-\psi(\|h(v, u)\|) \quad \forall v, u \in K .
$$

DEFINITION 2.7. A mapping $A: K \rightarrow X^{*}$ is called $h-\phi$ Lipschitzian if there exist mappings $\phi: \mathbb{R}^{+} \rightarrow \mathbb{R}^{+}$with $\phi(0)=0, h: K \times K \rightarrow K$ satisfying

$$
\langle A v-A u, h(v, u)\rangle \leq \phi(\|h(v, u)\|) \quad \forall v, u \in K
$$

In a similar way, we can define the $h-\phi$ monotonicity of the mapping $C$ : $K \rightarrow X^{*}$ with respect to the third argument of $M$.

DEFINITION 2.8. A mapping $A: K \rightarrow X^{*}$ is called hemicontinuous if the real function $t \rightarrow\langle A(x+t y), z\rangle$ is continuous on $[0,1]$ for any $x, y, z \in K$.

REMARK 2.9. For a suitable choice of $M, A, B, C, h, \phi$, and $\psi$, Definitions 2.5, 2.6, and 2.7 include many known definitions of various monotone mappings, relaxed monotone mappings, and Lipschitzian mappings as special cases (see $[1,3,4,6,7]$ and the references therein).

3. Main results. Our main results are as follows.

THeOrem 3.1. Let $X$ be a reflexive Banach space, $X^{*}$ its dual space, and $K$ a nonempty convex closed subset of $X$. Suppose that $M: X^{*} \times X^{*} \times X^{*} \rightarrow X^{*}$ is continuous, $h: K \times K \rightarrow K$ is linear with respect to the first argument and satisfies $h(x, x)=0$ for all $x \in K$, and $f: K \rightarrow \mathbb{R}$ is convex functional. Assume that $A, B, C: K \rightarrow X^{*}$ are continuous from line segments in $K$ to the weak topology of $X^{*}$ such that $A$ is $h-\phi$ monotone with respect to the first argument of $M, B$ is $h-\psi$ relaxed monotone with respect to the second argument of $M, C$ is $h-\omega$ monotone with respect to the third argument of $M$, and

$$
\lim _{t \rightarrow 0^{+}} \frac{\phi(t)-\psi(t)+\omega(t)}{t}=0
$$


Then, for each given $w \in X^{*}, u \in K$ is a solution of problem (2.1) if and only if $u \in K$ is a solution of the following problem: find $u \in K$ such that

$$
\begin{aligned}
& \langle M(A v, B v, C v)-w, h(v, u)\rangle+f(v)-f(u) \\
& \quad \geq \phi(\|h(v, u)\|)-\psi(\|h(v, u)\|)+\omega(\|h(v, u)\|) \quad \forall v \in K .
\end{aligned}
$$

Proof. Suppose that $u \in K$ is a solution of problem (2.1). Since $A$ is $h-\phi$ monotone with respect to the first argument of $M, B$ is $h-\psi$ relaxed monotone with respect to the second argument of $M$, and $C$ is $h-\omega$ monotone with respect to the third argument of $M$, it follows that

$$
\begin{aligned}
&\langle M(A v, B v, C v)-w, h(v, u)\rangle+f(v)-f(u) \\
&=\langle M(A v, B v, C v)-M(A u, B v, C v), h(v, u)\rangle \\
&+\langle M(A u, B v, C v)-M(A u, B u, C v), h(v, u)\rangle \\
&+\langle M(A u, B u, C v)-M(A u, B u, C u), h(v, u)\rangle \\
&+\langle M(A u, B u, C u)-w, h(v, u)\rangle+f(v)-f(u) \\
& \geq \phi(\|h(v, u)\|)-\psi(\|h(v, u)\|)+\omega(\|h(v, u)\|)
\end{aligned}
$$

for all $v \in K$. That is, (3.2) is satisfied.

Conversely, suppose that (3.2) holds. Let $v$ be an arbitrary point in $K$. Set $v_{t}=(1-t) u+t v$ for $t \in[0,1]$. Clearly, $v_{t} \in K$ and $v_{t}-u=t(v-u)$. Note that $h$ is linear with respect to the first argument and $h(x, x)=0$ for any $x \in K$. Thus,

$$
h\left(v_{t}, u\right)=(1-t) h(u, u)+\operatorname{th}(v, u)=\operatorname{th}(v, u) \quad \text { for any } v \in K
$$

Since $f$ is convex, by (3.2) and (3.4) we conclude that

$$
\begin{aligned}
t\left\{\left\langle M\left(A v_{t}, B v_{t}, C v_{t}\right)-w, h(v, u)\right\rangle+f(v)-f(u)\right\} \\
\geq\left\langle M\left(A v_{t}, B v_{t}, C v_{t}\right)-w, h\left(v_{t}, u\right)\right\rangle+f\left(v_{t}\right)-f(u) \\
\geq \phi\left(\left\|h\left(v_{t}, u\right)\right\|\right)-\psi\left(\left\|h\left(v_{t}, u\right)\right\|\right)+\omega\left(\left\|h\left(v_{t}, u\right)\right\|\right) \\
\quad \geq \varphi(t\|h(v, u)\|)-\psi(t\|h(v, u)\|)+\omega(t\|h(v, u)\|)
\end{aligned}
$$

for all $t \in(0,1]$ and $v \in K$. In view of (3.5), we get

$$
\begin{array}{r}
\left\langle M\left(A v_{t}, B v_{t}, C v_{t}\right)-w, h(v, u)\right\rangle+f(v)-f(u) \\
\geq \frac{\phi(t\|h(v, u)\|)-\psi(t|| h(v, u) \|)+\omega(t\|h(v, u)\|)}{t}
\end{array}
$$

for all $t \in(0,1]$ and $v \in K$. Since $M$ is continuous and $A, B, C$ are weakly continuous on segments of $K, M\left(A v_{t}, B v_{t}, C v_{t}\right)$ weakly converges to $M(A u, B u, C u)$ 
as $t \rightarrow 0^{+}$. It follows from (3.1) and (3.6) that

$$
\langle M(A u, B u, C u)-w, h(v, u)\rangle+f(v)-f(u) \geq 0 \quad \forall v \in K
$$

This completes the proof.

REMARK 3.2. Theorem 2.1 of Verma [7] and Theorem 1 of Browder [1] are particular cases of Theorem 3.1.

From Theorem 3.1, we immediately obtain the following result.

THEOREM 3.3. Let $X, X^{*}, K, h$, and $f$ be as in Theorem 3.1. Assume that $A, B, C: K \rightarrow X^{*}$ are hemicontinuous mappings such that $A$ is $h-\phi$ monotone, $B$ is $h-\psi$ Lipschitzian, $C$ is $h-\omega$ monotone, and (3.1) holds. Then, for any $w \in X^{*}, u \in K$ is a solution of problem (2.2) if and only if $u \in K$ is a solution of the following problem: find $u \in K$ such that

$$
\begin{aligned}
\langle A v & -B v+C v-w, h(v, u)\rangle+f(v)-f(u) \\
& \geq \phi(\|h(v, u)\|)-\psi(\|h(v, u)\|)+\omega(\|h(v, u)\|) \quad \forall v \in K .
\end{aligned}
$$

REMARK 3.4. Theorem 3.3 extends, improves, and unifies Lemma 1 of Carbone [3] and Lemma 1 of Siddiqi et al. [6].

THEOREM 3.5. Let $X, X^{*}, K, M, h, f, A, B, C, \phi, \psi$, and $\omega$ be as in Theorem 3.1. If $\phi, \psi$, and $\omega$ are continuous and $f$ is lower semicontinuous, then problem (2.1) has a solution. Furthermore, if the following conditions:

$$
\begin{aligned}
& h(u, v)=-h(v, u) \quad \forall u, v \in K, \\
& h(u, v)=0 \text { implies that } u=v, \\
& \phi(t)-\psi(t)+\omega(t)>0 \quad \forall t>0
\end{aligned}
$$

are fulfilled, then problem (2.1) has a unique solution.

Proof. Let the multivalued mappings $F, G: K \rightarrow 2^{K}$ be defined as

$$
\begin{gathered}
F v=\{u \in K:\langle M(A u, B u, C u)-w, h(v, u)\rangle+f(v)-f(u) \geq 0\}, \\
G v=\{u \in K:\langle M(A v, B v, C v)-w, h(v, u)\rangle+f(v)-f(u) \\
\geq \phi(\|h(v, u)\|)-\psi(\|h(v, u)\|)+\omega(\|h(v, u)\|)\}
\end{gathered}
$$

for all $v \in K$, respectively. Now, we claim that $F$ is a KKM mapping on $K$. If not, then there exist $\left\{u_{i}: 1 \leq i \leq n\right\} \subset K, \sum_{i=1}^{n} t_{i}=1, t_{i}>0$ for each $i \in\{1, \ldots, n\}$ 
and $u=\sum_{i=1}^{n} t_{i} u_{i} \notin \bigcup_{i=1}^{n} F u_{i}$. It follows that

$$
\begin{aligned}
0 & =\langle M(A u, B u, C u)-w, h(u, u)\rangle \\
& =\left\langle M(A u, B u, C u)-w, h\left(\sum_{i=1}^{n} t_{i} u_{i}, u\right)\right\rangle \\
& =\sum_{i=1}^{n} t_{i}\left\langle M(A u, B u, C u)-w, h\left(u_{i}, u\right)\right\rangle \\
& <\sum_{i=1}^{n} t_{i}\left(f(u)-f\left(u_{i}\right)\right) \\
& =f(u)-\sum_{i=1}^{n} t_{i} f\left(u_{i}\right) \\
& \leq 0
\end{aligned}
$$

which is a contradiction. Hence, $F$ is a KKM mapping.

Next, we claim that $F v \subset G v$ for all $v \in K$. Indeed, let $u \in F v$. Then, we infer that

$$
\begin{aligned}
&\langle M(A v, B v, C v)-w, h(v, u)\rangle+f(v)-f(u) \\
&=\langle M(A v, B v, C v)-M(A u, B v, C v), h(v, u)\rangle \\
&+\langle M(A u, B v, C v)-M(A u, B u, C v), h(v, u)\rangle \\
&+\langle M(A u, B u, C v)-M(A u, B u, C u), h(v, u)\rangle \\
&+\langle M(A u, B u, C u)-w, h(v, u)\rangle+f(v)-f(u) \\
& \geq \phi(\|h(v, u)\|)-\psi(\|h(v, u)\|)+\omega(\|h(v, u)\|)
\end{aligned}
$$

for all $v \in K$. That is, $u \in G v$. Thus, $G$ is a KKM mapping. It follows from Theorem 3.1 that

$$
\bigcap_{v \in K} F v=\bigcap_{v \in K} G v
$$

Since $f$ is lower semicontinuous and $h, \varphi, \psi$, and $\omega$ are continuous, therefore $G v$ is closed for any $v \in K$. Note that $K$ is bounded closed convex. It is clear that $K$ is weakly compact set in $X$. Hence, $G v$ is weakly compact set in $K$ since $G v \subset K$ for each $v \in K$. Lemma 2.2 and (3.13) ensure that

$$
\bigcap_{v \in K} F v=\bigcap_{v \in K} G v \neq \varnothing
$$

which implies that there exists $u \in K$ such that

$$
\langle M(A u, B u, C u)-w, h(v, u)\rangle+f(v)-f(u) \geq 0 \quad \forall v \in K .
$$

That is, problem (2.1) has a solution $u \in K$. 
Finally, we show the uniqueness of solution. Suppose that $v \in K$ is another solution of problem (2.1) with $v \neq u$. Then,

$$
\begin{aligned}
& \langle M(A u, B u, C u)-w, h(v, u)\rangle+f(v)-f(u) \geq 0, \\
& \langle M(A v, B v, C v)-w, h(u, v)\rangle+f(u)-f(v) \geq 0 .
\end{aligned}
$$

Thus, (3.9) and (3.16) yield

$$
\langle M(A u, B u, C u)-M(A v, B v, C v), h(v, u)\rangle \geq 0 .
$$

By (3.9) and (3.17), we infer that

$$
\begin{aligned}
0 \leq & \langle M(A u, B u, C u)-M(A v, B v, C v), h(v, u)\rangle \\
= & \langle M(A u, B u, C u)-M(A v, B u, C u), h(v, u)\rangle \\
& +\langle M(A v, B u, C u)-M(A v, B v, C u), h(v, u)\rangle \\
& +\langle M(A v, B v, C u)-M(A v, B v, C v), h(v, u)\rangle \\
\leq & -[\phi(\|h(v, u)\|)-\psi(\|h(v, u)\|)+\omega(\|h(v, u)\|)] \\
< & 0,
\end{aligned}
$$

which is a contradiction. This completes the proof.

As a consequence of Theorem 3.5, we have the following theorem.

THeOrem 3.6. Let $X, X^{*}, K, h, f, A, B, C, \phi, \psi$, and $\omega$ be as in Theorem 3.3. If $\phi, \psi$, and $\omega$ are continuous and $f$ is lower semicontinuous, then problem (2.2) has a solution. Furthermore, if (3.9) holds, then problem (2.2) has a unique solution.

REMARK 3.7. Theorem 3 of Siddiqi et al. [6] and Theorem 2.2 of Verma [7] are special cases of Theorem 3.6.

THeOrem 3.8. Let $X$ be a topological vector space, $X^{*}$ the topological dual space of $X, K$ a nonempty convex subset of $X, D$ a nonempty compact convex set in $K$, and the bilinear form $\langle\cdot, \cdot\rangle$ continuous. Suppose that $A, B, C: K \rightarrow X^{*}$, $M: X^{*} \times X^{*} \times X^{*} \rightarrow X^{*}$ are continuous and $f: K \rightarrow \mathbb{R}$ is convex lower semicontinuous functional. Assume that $h: K \times K \rightarrow K$ is linear with respect to the first argument and continuous with respect to the second argument. If, for each given $w \in X^{*}$, the following condition:

$$
\langle M(A u, B u, C u)-w, h(u, u)\rangle \geq 0 \quad \forall u \in K
$$

is satisfied and the set

$$
Y=\{v \in K:\langle M(A v, B v, C v)-w, h(u, v)\rangle+f(u)-f(v) \geq 0 \forall u \in D\}
$$

is compact, then problem (2.1) has a solution in $\mathrm{K}$. 
Proof. Set

$$
E=\{(u, v) \in K \times K:\langle M(A v, B v, C v)-w, h(u, v)\rangle+f(u)-f(v) \geq 0\}
$$

where $w$ is a given point in $K$. Condition (3.19) ensures that $(u, u) \in E$. For each $u \in K$, the set

$$
E_{u}=\{v \in K:\langle M(A v, B v, C v)-w, h(u, v)\rangle+f(u)-f(v) \geq 0\}
$$

is closed as can be easily seen from the continuity of $M, A, B, C,\langle\cdot, \cdot\rangle$, and $h$ relative to the second argument and lower semicontinuity of $f$. Now, we assert that, for each $v \in K$, the set

$$
E_{v}=\{u \in K:\langle M(A v, B v, C v)-w, h(u, v)\rangle+f(u)-f(v)<0\}
$$

is convex. Let $x, y \in E_{v}$ and $t, s>0$ with $t+s=1$. Then, we know that

$$
\begin{gathered}
\langle M(A v, B v, C v)-w, \operatorname{th}(x, v)\rangle+t f(x)-t f(v)<0, \\
\langle M(A v, B v, C v)-w, \operatorname{sh}(y, v)\rangle+s f(y)-s f(v)<0 .
\end{gathered}
$$

Note that $h$ is linear relative to the first argument and $f$ is convex. By virtue of (3.24), we infer that

$$
\begin{aligned}
&\langle M(A v, B v, C v)-w, h(t x+s y, v)\rangle+f(t x+s y)-f(v) \\
& \leq\langle M(A v, B v, C v)-w, \operatorname{th}(x, v)\rangle+t f(x)-t f(v) \\
&+\langle M(A v, B v, C v)-w, \operatorname{sh}(y, v)\rangle+s f(y)-s f(v) \\
&<0 .
\end{aligned}
$$

Since $K$ is convex, by (3.25) we obtain that $t x+s y \in E_{v}$. It follows from Lemma 2.3 that there exists $u \in K$ such that $K \times\{u\} \subset E$. This means that

$$
\langle M(A u, B u, C u)-w, h(v, u)\rangle+f(v)-f(u) \geq 0 \quad \forall v \in K .
$$

That is, $u \in K$ is a solution of problem (2.1). This completes the proof.

REMARK 3.9. In case where $w=0, M(x, y, z)=x$ for all $x, y, z \in X^{*}$, $h(x, y)=x-g y$ for all $x, y \in K, B x=C x=0$ for all $x \in K$, and $A: K \rightarrow X^{*}$ and $g: K \rightarrow K$ are continuous, Theorem 3.8 reduces to [4, Theorem 2].

From Theorem 3.8, we have the following theorem.

THEOREM 3.10. Let $X, X^{*},\langle\cdot, \cdot\rangle, A, B, C, M, f$, and $h$ be as in Theorem 3.8, where $K$ is a nonempty compact convex set in $X$. If (3.19) holds, then problem (2.1) has a solution in $K$.

REMARK 3.11. If $\left\langle X, X^{*}\right\rangle$ is a dual system of locally convex spaces, Theorem 3.10 reduces to a result which extends [3, Theorem 1] and [6, Theorem 4]. 
ACKNOWLEDGment. This research was financially supported by Changwon National University in 2002.

\section{REFERENCES}

[1] F. E. Browder, Nonlinear monotone operators and convex sets in Banach spaces, Bull. Amer. Math. Soc. 71 (1965), 780-785.

[2] A. Carbone, Variational inequalities and fixed points, Indian J. Math. 36 (1994), no. 3, 193-200.

[3] _ A note on a variational inequality, Indian J. Pure Appl. Math. 27 (1996), no. $12,1225-1228$.

[4] _ A note on complementarity problem, Int. J. Math. Math. Sci. 21 (1998), no. 3, 621-623.

[5] K. Fan, A generalization of Tychonoff's fixed point theorem, Math. Ann. 142 (1960/1961), 305-310.

[6] A. H. Siddiqi, Q. H. Ansari, and K. R. Kazmi, On nonlinear variational inequalities, Indian J. Pure Appl. Math. 25 (1994), no. 9, 969-973.

[7] R. U. Verma, Nonlinear variational inequalities on convex subsets of Banach spaces, Appl. Math. Lett. 10 (1997), no. 4, 25-27.

Zeqing Liu: Department of Mathematics, Liaoning Normal University, Dalian, Liaoning 116029, China

E-mail address: zeqing7iu@sina.com.cn

Jeong Sheok Ume: Department of Applied Mathematics, Changwon National University, Changwon 641-773, Korea

E-mail address: jsume@sarim. changwon.ac.kr

Shin Min Kang: Department of Mathematics and Research Institute of Natural Science, Gyeongsang National University, Chinju 660-701, Korea

E-mail address: smkang@nongae.gsnu.ac. kr 


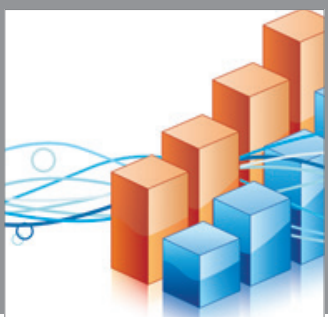

Advances in

Operations Research

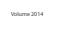

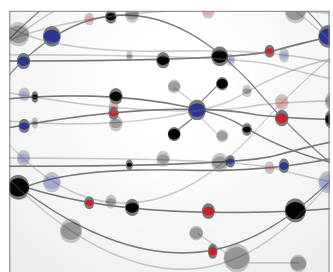

\section{The Scientific} World Journal
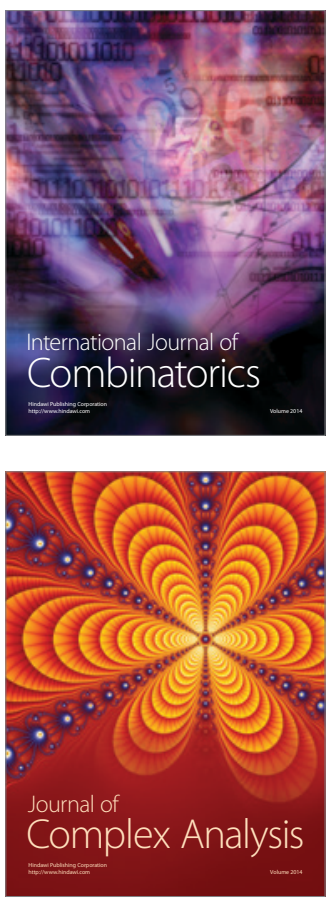

International Journal of

Mathematics and

Mathematical

Sciences
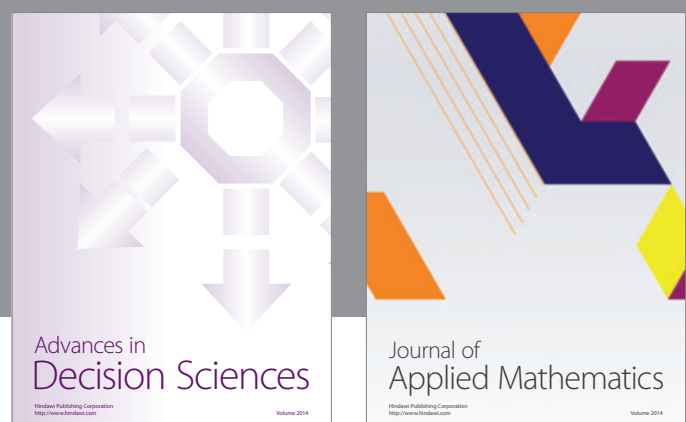

Journal of

Applied Mathematics
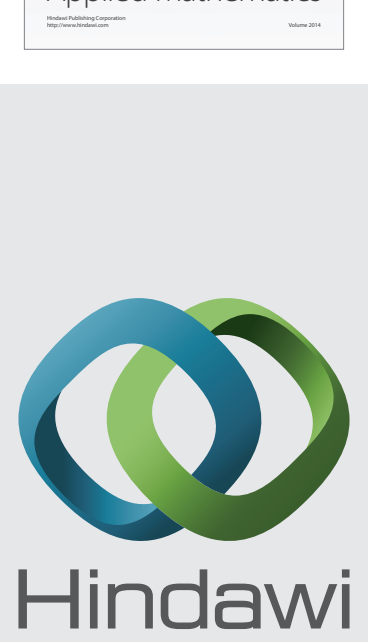

Submit your manuscripts at http://www.hindawi.com
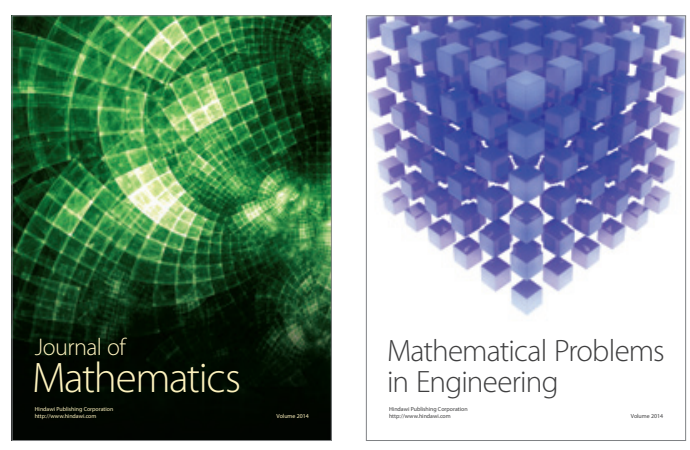

Mathematical Problems in Engineering
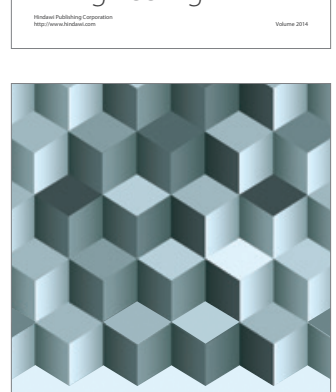

Journal of

Function Spaces
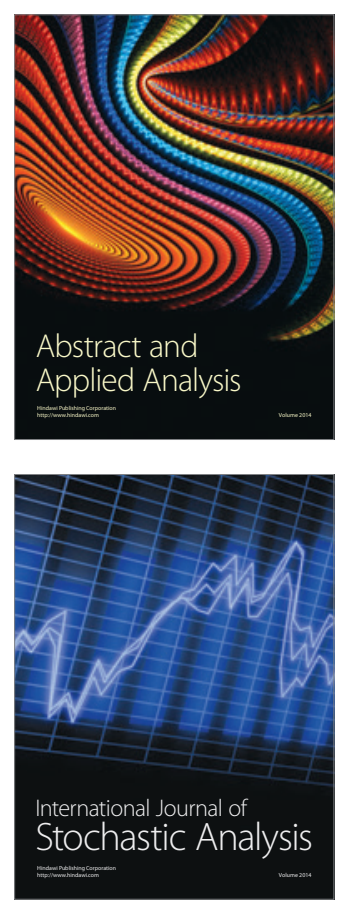

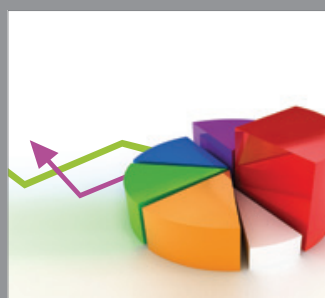

ournal of

Probability and Statistics

Promensencen
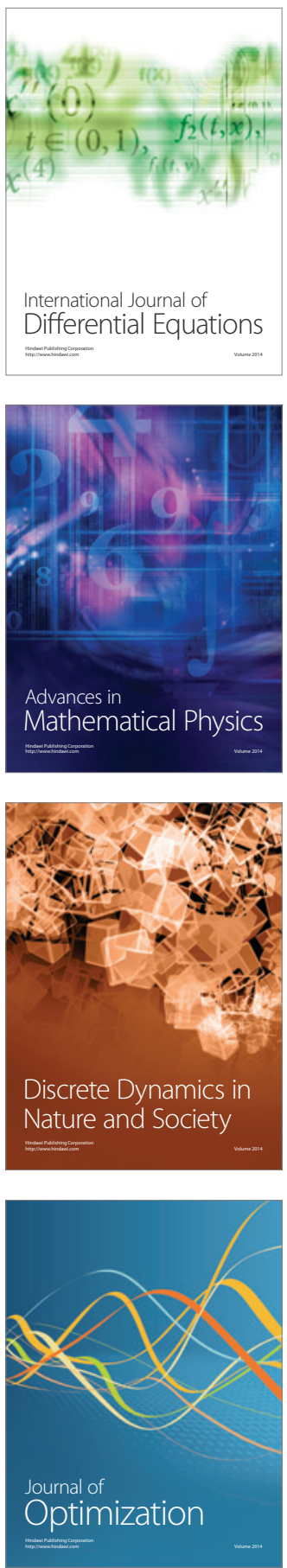